\title{
Path of awareness for the construction of new personal meanings. Qualitative analysis of the Purpose in Life (PIL) Questionnaire in an oncologic Italian population
}

\author{
Loredana Buonaccorso', Gianfranco Martucci ${ }^{2}$, Guido Miccinesi ${ }^{3}$, Alice Maruelli ${ }^{4}$, Carla Ripamonti \\ 1. Psycho-oncology Unit, Scientific Direction, Azienda UsI - IRCSS Reggio Emilia \\ 2. Italian League for the Fight Against Cancer (LILT) Reggio Emilia \\ 3. Clinical Epidemiology Unit, ISPRO-Istituto per lo Studio, la Prevenzione e la Rete Oncologica, Florence \\ 4. Psychology Unit, LILT and Center for Oncological Rehabilitation-CERION Florence \\ 5. Oncology-Supportive Care Unit, Department Onco-Hematology, Fondazione IRCCS, National Cancer Instiute, Milan
}

Introduction. The search of meaning, as well as the possibility to find a purpose in the face of suffering associated with oncological disease, influences the well-being of patients. Patients can also experience positive aspects from their illness it might generate new personal and existential forms of meaning, as a consequence of an improved awareness of problems that might have been already present in their daily life, but that they were pushed to face by their illness $(1,2)$. Objective. We investigated the meaning that cancer patients in active therapy and/or rehabilitation give to their illness and which resources they put in practice to build new experiences of meaning and a future perspective.

Methods. The data were collected from patients participating to a Big cross-sectional multicenter Project conducted in order to validate the Italian version of questionnaires created to assess existential dimensions of the patients' experience (dignity, hope) and to study the correlation between them and the symptoms of the patients as assessed by the Italian version of ESAS, during the first six months of 2011 (3). For the present study, we discussed the thematic analysis of the answers to open questions of part B and C of the Purpose In Life Questionnaire (PIL) in 158 consecutive cancer patients with nonadvanced cancer (no metastases) according to clinical data and stage of the disease reported in the patients' clinical chart.
The PIL is an attitude scale developed by Crumbaugh and Maholick, based on Frankl's logotherapy. It indicates the degree to which meaning and purpose in life have been attained by the respondents. It consists of 20 statements, each rated on a seven-point verbal scale.

The PIL also includes a qualitative section that consists of 13 sentence completion items and a paragraph composition section concerning future goals and past meaningful experiences, which are most interesting in a therapeutic context (4).

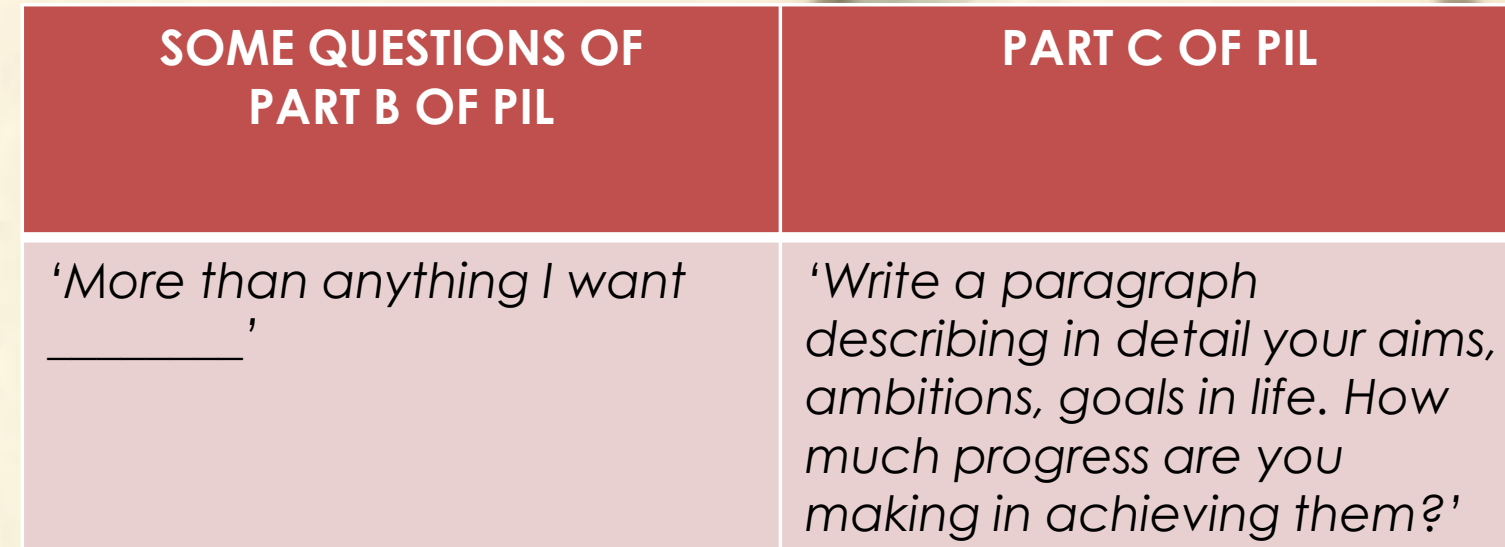

'The whole purpose of my life

'Death is

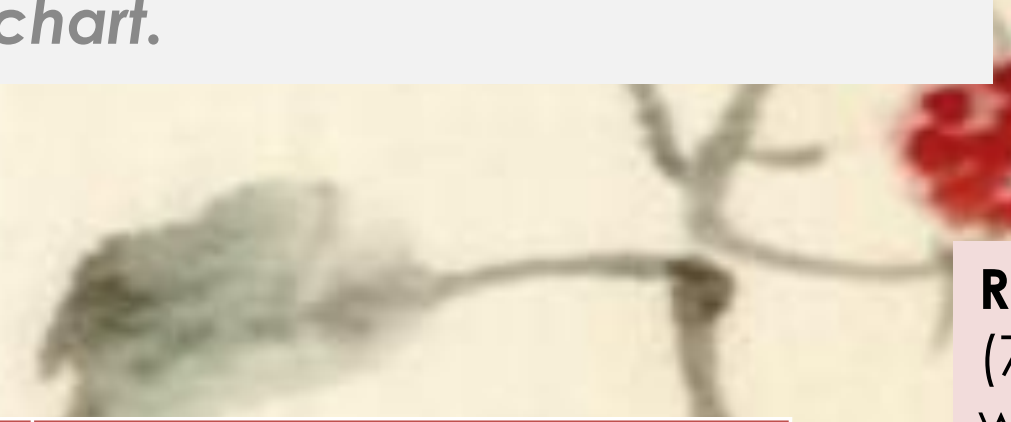

RESULTS. Most of the patients were male (60\%), lived with a partner $(73 \%)$, with an average age of 65 , had high school education $(45 \%)$, were retired (44\%) and churchgoer (45\%), with breast cancer (35\%), in treatment or rehabilitation $(75.3 \%)$.

The data show the recurrence of a strong sense of positivity, present in the majority of the interviews. In particular, this positivity is showed by the use of words associated frequently to self-consciousness and selfevaluation, desire of happiness and desire of contributing to the good of significant others and of taking care of your own life in order to give a positive contribution.

C147: ['Living your daily life at the fullest, let go the past, the dead, find myself, living in the present, search for beauty and lightness, loving and saying it out loud'].

C166: ['One of my purposes, maybe the main one, is not to waste this vital energy, that I now feel inside of me, for pointless things. I want to appreciate what I have. I would like to help women that went under surgery for breast cancer. The purpose of my life is to know myself more and more, to get in contact with my perception. I tried not to 1 take anything for granted and I focus more on myself'].

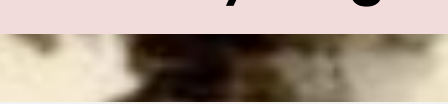

In our analysis the illness seen as an opportunity is the main theme that seems to orient all the other categories and sub-themes: 77 patients over 124 lived the illness as an occasion to grow and confront themselves. From the positive meanings of the answers that we find in all these questions the necessity of giving a purpose to existence emerges, even when it seems to be more under threat. 